\title{
Global and local conductivity in percolating crosslinked carbon black/epoxy-amine composites
}

\author{
A. Alekseev ${ }^{1,4}$, T. H. Wu ${ }^{1}$, L. G. J. van der Ven², R. A. T. M. van Benthem ${ }^{2,3}$, and \\ G. de With ${ }^{1,2, *}$
}

${ }^{1}$ Laboratory of Materials and Interface Chemistry, Department of Chemical Engineering and Chemistry, Eindhoven University of Technology, PO Box 513, 5600 MB Eindhoven, The Netherlands

${ }^{2}$ Laboratory of Physical Chemistry, Department of Chemical Engineering and Chemistry, Eindhoven University of Technology, PO Box 513, 5600 MB Eindhoven, The Netherlands

${ }^{3}$ DSM Materials Science Center, PO Box 18,6160 MD Geleen, The Netherlands

${ }^{4}$ National Research University "MIET", Moscow 124498, Russia

Received: 13 December 2019

Accepted: 4 April 2020

Published online:

20 April 2020

(C) The Author(s) 2020

\begin{abstract}
Conductive particle-filled polymer composites are promising materials for applications where both the merits of polymer and conductivity are required. The electrical properties of such composites are controlled by the particle percolation network present in the polymeric matrix. In this study, the electrical properties of crosslinked carbon black-epoxy-amine (CB-EA) composites with various $\mathrm{CB}$ concentrations are studied at room temperature as a function of the AC frequency $f$. A transition at critical frequency $f_{\mathrm{c}}$ from the DC plateau $\sigma_{\mathrm{DC}}$ to a frequency-dependent part was observed. Conductivity mechanisms for $f>f_{\mathrm{c}}$ and $f<f_{\mathrm{c}}$ were investigated. By considering the fractal nature, conduction for $f>f_{\mathrm{c}}$ was verified to be intra-cluster charge diffusion. For $f<f_{\mathrm{c}}$, with the assistance of conductive atomic force microscopy (C-AFM), the conduction behavior of individual clusters can be observed, revealing both linear and nonlinear $I-V$ characteristics. By combining microtoming and C-AFM measurements, 3D reconstructed images offer direct evidence that the percolating network of these materials consists of both a low-conductivity part, in which the charge transports through tunneling, and a high-conductivity part, which shows ohmic electrical properties. Nevertheless, for these CB-EA composites, the presence of these non-ohmic contacts still leads to Arrhenius-type behavior for the macroscopic conductivity.
\end{abstract}




\section{Introduction}

Epoxy-amine resins are widely applied in various fields where easy processing, low water uptake, excellent adhesion and good corrosion protection are needed [1-4]. Such polymer systems are generally electrically insulating, having a volume resistivity from $10^{12}$ to $10^{15} \Omega \mathrm{cm}$ [5]. By incorporating conductive particles, i.e., carbon blacks (CB) [6-9], carbon nanotubes $[9,10]$ or metallic fillers [11], the material can be altered from an insulator to a (semi)conductor, resulting in a broader range of application possibilities. However, the insulating-to-conductive transition is generally not a gradual one [6-13]. Instead, it is characterized by an abrupt increase with several orders of magnitude at a certain particle loading [14]. Above this critical concentration, the percolation threshold, a phase consisting of an insulating polymeric matrix and a (semi)conductive particle network exist. Electrons are transported via conductive pathways in these networks.

To deal with such disordered systems in which long-range connectivity suddenly appears, percolation theory has been extensively applied [14, 15]. Based on this theory, the conductivity behavior follows a universal rule when the particle concentration $p$ is close to the percolation threshold $p_{c}$, i.e., for $\left|p-p_{\mathrm{c}}\right| \ll 1[14,15]$, given by

$\sigma_{\mathrm{DC}} \propto\left(p-p_{\mathrm{c}}\right)^{t}$

with $\sigma_{\mathrm{DC}}\left(\mathrm{S} \mathrm{cm}^{-1}\right)$ the DC conductivity and $t$ a critical exponent. A Monte Carlo simulation for three-dimensional random percolation yielded $2.0 \pm 0.2$. However, such a simulation does not take into account specific interactions and reactions. In real systems, many experiments displayed results in which $t$-values higher than 2 were found, implying a different type of conduction mechanism that is more sensitive to increasing particle concentration than predicted by conventional percolation theory.

One way to study material properties is $3 \mathrm{D}$ imaging by AFM, a relatively new area of research which started 20 years ago [16]. The use of this technique requires controllable, subsequent removal of thin surface layers, since AFM is surface characterization method. Different methods can be used for the removal of material from surfaces, e.g., plasma or wet etching, microtoming and scratching by the AFM probe [17]. After removal of each slice, the surface is measured by a suitable AFM method, where after the set of 2D AFM images obtained can be used to construct a 3D image. An advantage of this technique is the possibility to construct 3D images of different properties by using different AFM modes, in particular the distribution of local electrical properties, such as the current and surface potential [18, 19]. Here, we use combination of AFM and ultramicrotomy for the $3 \mathrm{D}$ reconstruction of the conductive network inside a CB-EA composite by measurements of the current distribution on sample surfaces with conductive AFM (C-AFM). The lateral $(x, y)$ resolution of the reconstructed volume structure is determined by resolution of the AFM, and z-resolution is determined by accuracy of microtome cutting step, which can be as small as $10 \mathrm{~nm}$.

In previous work, CB-epoxy-amine (CB-EA) composites were prepared and analyzed [20]. The clusters were demonstrated to be fractal and influenced by the crosslinking process, resulting in a fractal dimension $d_{\mathrm{f}}=1.66$ for high-temperature curing and $d_{\mathrm{f}}=1.56$ for room-temperature curing, both notably smaller than 1.85 for diffusion-limited cluster aggregation (DLCA) mechanism. Moreover, the percolation threshold for this composite was found at $\mathrm{CB}$ concentration $0.24 \mathrm{vol} \%$, and the corresponding $t$ value was $2.9 \pm 0.14$, considerably deviating from the value $2.0 \pm 0.2$ obtained from Monte Carlo simulation for three-dimensional (3D) random percolation. In order to obtain a deeper understanding of the electrical conduction of these materials, in this study AC conductivity measurements were done as a function of $\mathrm{CB}$ concentration, and the transition from the DC plateau to frequency-dependent $\mathrm{AC}$ behavior was determined. Furthermore, using C-AFM combined with ultramicrotoming, the conductivity behavior of individual percolating pathways was, to our knowledge, revealed for the first time.

\section{Experimental}

Preparation and characterization of the CBepoxy-amine nanocomposites

The preparation of CB-epoxy-amine composites with $\mathrm{CB}$ concentration ranging from 0.25 to $1.25 \mathrm{vol} \%$ has been described in detail in our previous work [20]. In brief, carbon black particles (KEC-600J, AkzoNobel) were ground into a fine powder in a mortar and dried at $50{ }^{\circ} \mathrm{C}$ for 5 days under vacuum. Afterward, a 
master batch with $2 \mathrm{vol} \%$ of CB was prepared by dispersing these particles in a bisphenol A-based epoxy resin (Epikote 828, Resolution Nederland BV) for $8 \mathrm{~h}$ at $6000-15000 \mathrm{rpm}$ by using a disk agitator (Dispermat CA40-C1, VMA) until a homogeneous dispersion was obtained. During this process, the temperature remained below $80{ }^{\circ} \mathrm{C}$. No additive was used in the whole process. To parts of this dispersion, epoxy resin and crosslinker (Jeffamine D230, Huntsman) were added to obtain the desired CB concentrations (0.25-1.25 vol\%) at an epoxy/NH molar ratio of 1.2/1. Mechanical stirring was then performed for $1 \mathrm{~min}$. Immediately after applying on substrates by using a quadruple applicator (Erichsen $\mathrm{GmbH} \& \mathrm{Co}$. $\mathrm{KG})$, the samples were placed into an oven. The curing cycle is at $20^{\circ} \mathrm{C}$ for 4 days and then a postcure at $100{ }^{\circ} \mathrm{C}$ for $4 \mathrm{~h}$. Differential scanning calorimetry (DSC) indicates a degree of conversion of 0.99, while transmission electron microscopy (TEM) shows that the particles are distributed homogeneously with a fractal dimension $d_{\mathrm{f}}=1.56$ [20].

\section{AC conductivity measurement}

For each composite, free-standing films were made and from samples with a thickness of $40-60 \mu \mathrm{m}$, pieces of $15 \times 15 \mathrm{~mm}$ were cut. Silver paint (Fluka) was applied on both faces of the films as electrodes as well to reduce the contact resistance. The conductivity $\sigma_{\mathrm{AC}}$ was measured at room temperature as a function of frequency $f$ using an impedance analyzer (EG\&G, Model 283) equipped with a frequency response detector (EG\&G, Model 1025) in the frequency range from $10^{-2}$ to $10^{6} \mathrm{~Hz}$.

\section{Temperature-dependent DC conductivity measurement}

Measurement of $\sigma_{\mathrm{DC}}$ as a function of temperature from 145 to $345 \mathrm{~K}$ was performed on a sample with $1.25 \mathrm{vol} \%$ CB. A sample with a thickness of $0.25 \mathrm{~mm}$ was cut into $2 \times 0.8 \mathrm{~cm}^{2}$, with silver paint (Fluka) applied on both sides in order to reduce the contact resistance. The measurement was done by placing the sample into a brass cell, equipped with temperature control and a two-point measurement set (DVM 890, Velleman), placed in a liquid nitrogen bath. For each measurement, the system was set to a fixed temperature divided in approximately 20 intervals over the temperature range used, allowed to equilibrate and kept constant during data acquisition.

\section{C-AFM analysis}

Two samples with CB concentrations (1 and $1.25 \mathrm{vol} \%)$, well above the percolation threshold, were used for conductive AFM (C-AFM) measurement. Conductive gold-coated cantilevers NSC36/ $\mathrm{Cr}-\mathrm{Au}$ (Micromash) were applied for conductivity measurements using an AFM equipped with an ultramicrotome (Ntegra Tomo, NT-MDT Co.). Both topography and current distribution are measured at the same time in contact mode with C-AFM. The sample is always grounded, and a voltage is applied to the tip. An oscillating diamond knife (Diatome) was used for cutting. Alternate microtome cutting and measurements of the same area by C-AFM result in a stack of AFM images having a $z$-interdigitation (thickness of each section) of $20 \mathrm{~nm}$. This array of scans has been aligned in one 3D image by using a simple procedure for which a more detailed description can be found in [18].

\section{Results and discussion}

\section{AC conductivity}

The AC conductivity $\sigma_{\mathrm{AC}}$ is derived from the complex impedance data [21] using

$\sigma_{\mathrm{AC}}=\left[Z^{\prime} /\left(Z^{\prime 2}+Z^{\prime \prime 2}\right)\right] \times(d / A)$

where $Z^{\prime}$ and $Z^{\prime \prime}$ are the real and imaginary parts of the impedance, respectively, $d$ the sample thickness, and $A$ the sample area. The conductivities as a function of frequency $f$ for CB-EA composites with different $\mathrm{CB}$ concentrations are plotted in Fig. 1a, in which an increase in intercept values as well as an extension of the plateau for each sample can be observed with increasing CB content. With increasing $f$, a $f$-independent to $f$-dependent transition occurs at a critical $f_{\mathrm{c}}$. In the low $-f$ region (in this study $10^{0}-10^{-2}$ $\mathrm{Hz}$ ), where the AC conduction mechanism is the same as that for DC conduction, the intercept value is generally considered to represent the DC conductivity $\sigma_{\mathrm{DC}}$.

For such a system with a conductive percolation network, the transition of conductivity from the DC plateau to frequency-dependent $\mathrm{AC}$ behavior is 

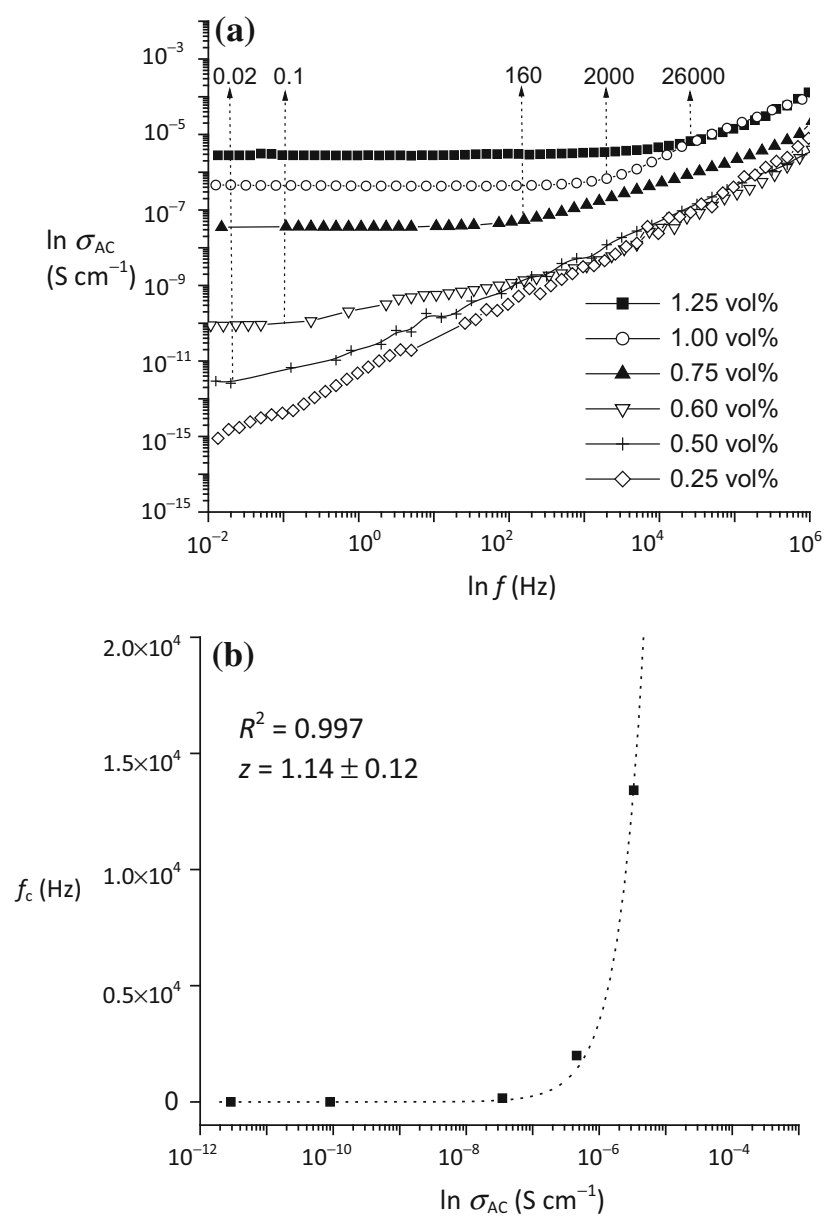

Figure 1 a The $\mathrm{AC}$ conductivity as a function of scanning frequency for $\mathrm{CB}-\mathrm{EA}$ samples with $\mathrm{CB}$ concentrations ranging from 0.25 to $1.25 \mathrm{vol} \%$; $\mathbf{b}$ the plot of $f_{\mathrm{c}}$ versus $\ln \boldsymbol{\sigma}_{\mathrm{AC}}$ as well as the fit based on Eq. (3).

usually described by a relationship between $f_{\mathrm{c}}$ and $\sigma_{\mathrm{DC}}$ given as [22]

$f_{\mathrm{c}} \propto \sigma_{\mathrm{DC}}^{z}$

The critical frequency $f_{\mathrm{c}}$ for all samples, except the $0.25 \mathrm{vol} \%$ samples, was taken as the frequency at the intersection of the lines describing the $\sigma_{\mathrm{DC}}$ plateau and the (approximately) linear increase (Fig. 1a), and $z=1.14$ was obtained by fitting the $f_{\mathrm{c}-}-\sigma_{\mathrm{DC}}$ data according to Eq. (3) (Fig. 1b). To verify the validity of this value for our system, we note that percolation theory $[14,22]$ at the vicinity of $p_{\mathrm{c}}$ provides

$\xi \propto\left(p-p_{\mathrm{c}}\right)^{-0}$

where $v$ is a critical exponent and $\xi$ denotes the percolation correlation length, which can be regarded as the largest finite size of clusters possessing a fractal structure. Below this length scale, the clusters show self-similarity, and above, they can be considered as Euclidean. If the $f$-dependent behavior in our system is caused by the presence of fractal structures, the critical frequency $f_{\mathrm{c}}$ can be associated with the frequency $f_{\xi}$ associated with the correlation length $\xi$, so that

$f_{\mathrm{c}}=f_{\xi} \propto \xi^{-d_{\mathrm{rw}}} \propto\left|p-p_{c}\right|^{v d_{\mathrm{rw}}}$

where $d_{\mathrm{rw}}$ is the effective critical exponent for a random walk on a fractal structure. A value $4.17 \pm 0.08$ $\left(R^{2}=0.9999\right)$ was obtained for $v d_{\mathrm{rw}}$ by fitting using Eq. (5).

By inserting the known value $v=0.8[22,23]$, we obtain $d_{\mathrm{rw}}=5.21$. Inserting the fractal dimension $d_{\mathrm{f}}$ $=1.56$, as obtained before for the same composite [20], in the relation $[15,24] z=d_{\mathrm{rw}} /\left(d_{\mathrm{rw}}-d_{\mathrm{f}}+1\right)$, the value $z \cong 1.12$ results. This value is in good agreement with the experimental fitted value $z=1.14$ mentioned before, indicating that the assumption of $f_{c}=f_{\xi}$ is valid, and the $f$-dependent behavior in this composite is dominated by intra-cluster charge transport in fractal objects. Notice that the $z$-value 1.14 is smaller and closer to unity when compared with other values reported, namely 1.67 [22] and 1.35 [25]. Further note that the relation [14] $d_{\mathrm{rw}-}$ $=2+(t-\beta) / v$ holds, where $\beta$ is the critical exponent for the percolation probability $P \sim\left(p-p_{\mathrm{c}}\right)^{\beta}$. A reasonable estimate for $\beta$ is 0.4 [14] which, together with $v=0.8[22,23]$, yields $t=3.0$, consistent with the experimental value $t=2.9$. Based on the established relation $f_{\mathrm{c}}=f_{\xi}$, the deviation between $t=2.9$ experimentally and $t=2.0$ from simulations implies that another conduction mechanism, offered by clusters with a smaller $\xi$-value than predicted by percolation theory, contributes. Jäger et al. suggested that this additional conductivity is due to the electron tunneling through the gaps between clusters [24], but direct evidence of charge tunneling on fractal percolating clusters was still missing.

\section{Conductive atomic force microscopy (C- AFM) analysis}

To investigate the conductivity behavior of percolation clusters, C-AFM was utilized [18]. The topography of samples with 1 and $1.25 \mathrm{vol} \% \mathrm{CB}$ is shown in Fig. 2a and c, respectively, in which the CB particles, which are harder than the polymeric matrix, can be identified from the contrast as the brighter spots. (The vertical lines are caused by microtome cutting.) The 
corresponding current distribution images of the same area are shown in Fig. $2 b$ and d, respectively, where the different colors represent different current densities, as indicated by the color bar on the side. The average current calculated from the C-AFM images is almost independent of the tip-sample load (within the load range changed by a factor of 3), which means that contact resistance has only a small influence on measured current and the current is determined by the bulk properties. Note that many particles, especially those inside the circles shown in Fig. $2 a$ and c, cannot be found in Fig. $2 b$ and $d$, respectively, indicating that the particles missing in the current images are not contributing to the longrange conduction. In addition, with increasing $\mathrm{CB}$ concentration, it can be observed from Fig. $2 b$ and $d$ that more clusters display higher conductivity levels (shifting to red color). Such an effect should not occur in a composite where the conduction is mainly contributed by ohmic contacts between clusters, which are expected to display an on-off behavior only.

Figure $2 \mathrm{~g}$ and $\mathrm{h}$ shows the topography and the corresponding current distribution of the same area at another spot for a 1\% CB-EA sample. The difference between Fig. $2 b$ and h clearly indicates the large variations that occur locally in such a close-to-thepercolation threshold sample. The average current $I_{\text {ave }}$ over the scanned area as a function of applied voltage for the complete $10 \times 10 \mu \mathrm{m}^{2}$ image given in Fig. $2 \mathrm{f}$ shows that averaging over such an area already yields already linear $I-V$ behavior, albeit with a different slope for different spots (keep in mind that $I_{\text {ave, }}$ figuratively speaking, represents a $10 \times 10 \mu \mathrm{m}^{2}$ tip, as compared to a single point measurement with a typical tip contact area of $75-300 \mathrm{~nm}^{2}$, but that direct comparison with a $10 \times 10 \mu^{2}$ electrode result is not straightforward). The values for $I_{\text {ave }}$ for the two different spots of the 1\% CB-EA sample are $0.017 \mathrm{nA}$ and $0.076 \mathrm{nA}$, respectively, while the average current for the $1.25 \%$ CB-EA sample is $0.14 \mathrm{nA}$. For macroscopic measurements [20], one can expect a difference of a factor $2-4$ between $1 \%$ and $1.25 \%$ CBEA samples. Thus, a direct comparison of current data from micrometer-sized areas with macroscopic data is inappropriate, as relatively large variations of the average current over small areas are expected.

To confirm the presence of non-ohmic contacts, conductivity measurements were performed for individual clusters of the $1.25 \mathrm{vol} \% \mathrm{CB}$ sample, as indicated by numbers 1,2 and 3 in Fig. $2 d$, respectively, with the corresponding $I-V$ characteristics displayed in Fig. 2e. Cluster 1 in Fig. 2d shows the highest current densities, and a linear $I-V$ curve, indicating that a long-range conductive pathway formed by physical contact between $\mathrm{CB}$ particles has been built up, resulting in ohmic electrical behavior. Notice that the linear $I-V$ behavior of cluster 1 is only valid for the path from the scanning tip to the electrode and is not necessarily representative for the whole composite. Indeed, clusters 2 and 3 show a lower conductivity as well as nonlinear $I-V$ behavior, strongly suggesting electrical tunneling along the conductive pathway.

To investigate the conductive pathways inside a sample volume, a 3D reconstruction of the conductivity was made in the direct surroundings of clusters 1, 2 and 3. Figure 3a shows the results, in which clusters with similar color have similar conduction behavior. The dark-green parts are clusters in deeper layers that do not form conductive pathways with the clusters appearing on the surface. As illustrated for clusters 1, 2 and 3 before, a color change from pink/ white to blue indicates changes of current values from high to low, and in many cases changes of the conduction mechanism from ohmic to tunneling.

In Fig. 3b, several transitions between low- and high-conductivity clusters within one conductive pathway can be observed inside the sample volume. Such a transition indicates that the cluster possessing the lower conductivity is the bottleneck for charge transport along this conductive pathway, thus contributing to the nonlinearity of the overall $I-V$ characteristic. Once the low conductivity parts were removed by microtoming, the remaining conductive pathway showed a higher conductivity level as well as linear $I-V$ behavior. Similar results were also obtained for multiwalled carbon nanotubes/PS (MWNT/PS) composites [18] and graphene/PS composites [19]. The low-to-high conductivity transition in a single conductive pathway has been detected here for the first time. This has become possible due to small size of the filler particles, which implies a large amount of connections within a short distance and thus a higher probability to find a junction within the reconstructed volume. In contrast to conventional percolation theory, the percolation network formed in this material is composed of both highly and weakly conductive clusters. Hence, before a percolation network is formed through physical contact, electron tunneling can take place in some 

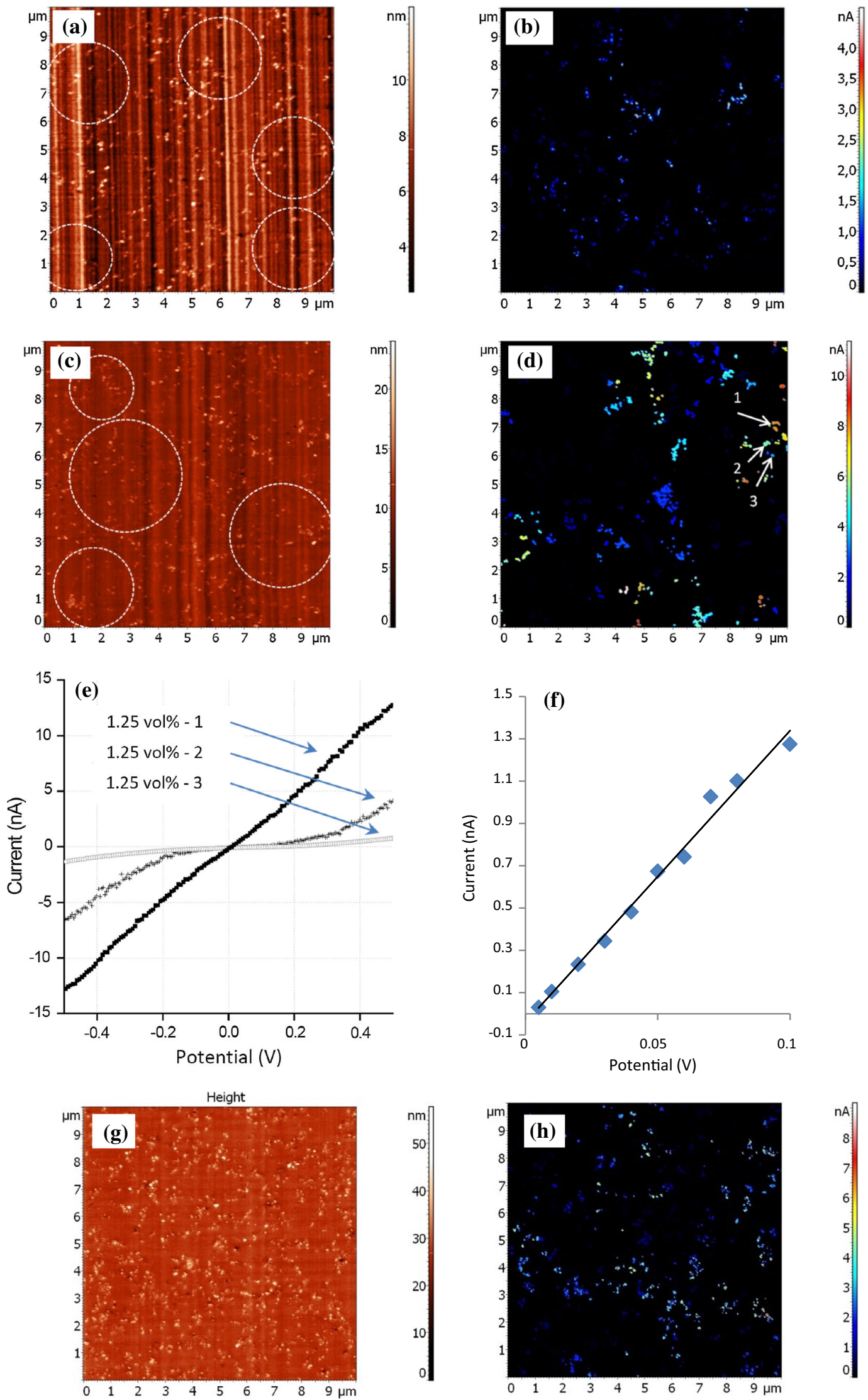
4 Figure 2 a and c Topography of CB-EA samples with 1 and $1.25 \mathrm{vol} \% \mathrm{CB}$, respectively; $\mathbf{b}$ and $\mathbf{d}$ the corresponding current distribution images; e the $I-V$ curves as measured for clusters 1,2 and 3, as indicated in Fig. 2d; $\mathbf{f} I_{\text {ave }}-V$ curve for the $10 \times 10 \mu \mathrm{m}^{2}$ area shown in $\mathbf{g}$ and $\mathbf{h} ; \mathbf{g}$ topography of a 1 vol\% CB-EA sample at a different spot; $\mathbf{h}$ the current distribution corresponding to $\mathbf{g}$

parts of the network, resulting in an increased overall conductivity, consistent with the $t$ and $z$ values as observed for DC and AC conductivity, respectively. The C-AFM images directly visualize the high-conductivity paths, which provide conductivity through the whole sample. These high-conductivity paths are surrounded by low-conductivity clusters, which are

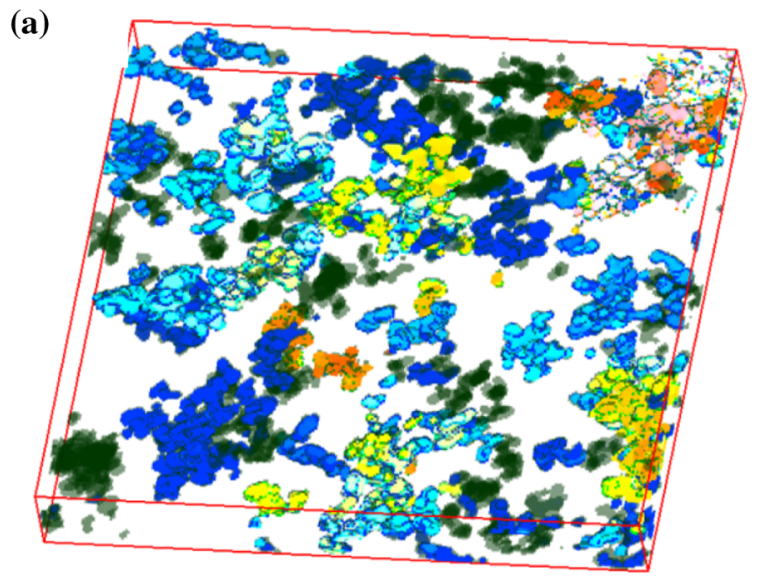

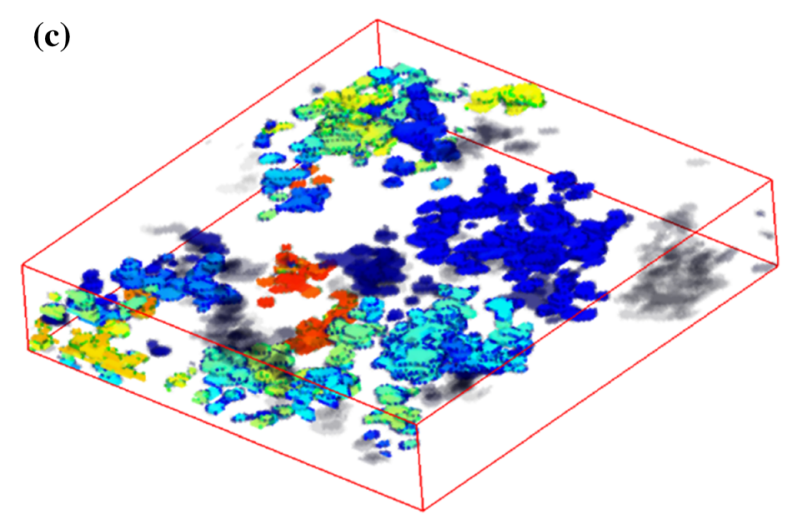

connected to the high-conductivity paths by tunneling contacts.

An attempt to provide additional evidence results from temperature-dependent DC conductivity measurements was made. For tunneling, the temperature dependence of $\sigma_{\mathrm{DC}}$ can be modeled by fluctuationinduced tunneling (FIT) theory [26] including contributions from thermal and voltage fluctuations in tunneling and given by

$\sigma_{\mathrm{DC}} \propto \exp \left[-T_{1} /\left(T+T_{0}\right)\right]$

Here, $T_{0}$ is the temperature that can be viewed as the temperature above which fluctuation effects become significant and $T_{1}$ is a measure of the energy required to move an electron across the insulating

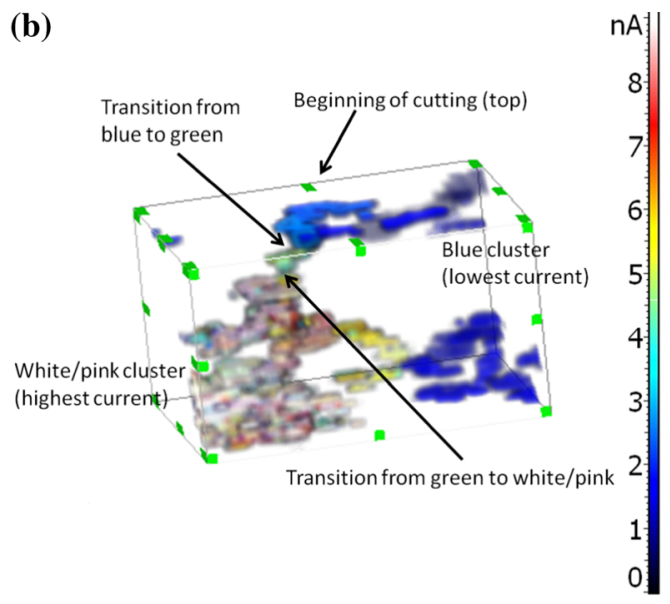

(d)

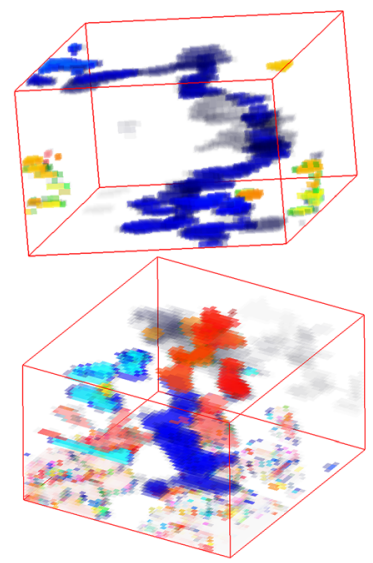

Figure 3 a 3D reconstructed image of a 1.25 vol\% CB-EA sample taken from the area where the clusters 1,2 and 3 are located (Fig. 2d). A microtome $z$-step of $20 \mathrm{~nm}$ was used, and 28 C-AFM images were obtained to reconstruct a volume of $4.5 \times 4.5$ $\times 0.54 \mu \mathrm{m}^{3}$. b A zoom-in 3D image of conductive clusters obtained from a, showing the transitions between clusters with high and low conductivity. c 3D reconstructed image of another 1.25 vol\% CB-EA sample; $\mathbf{d}$ A zoom-in 3D image of conductive clusters obtained from c, showing, respectively, a pathway with approximately constant conductivity (top) and a cluster with rapidly changing conductivity (bottom). 


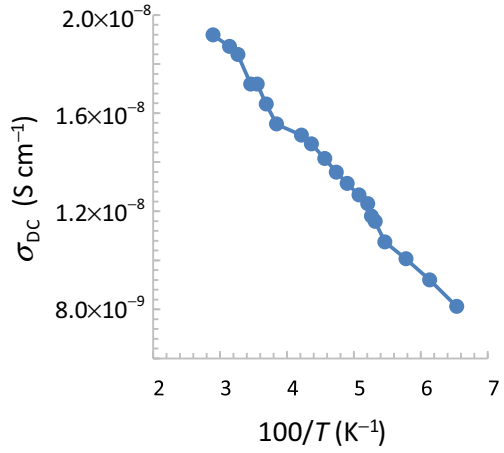

Figure 4 Arrhenius plot for a 1.25 vol\% CB-EA sample of the conductivity $\sigma_{\mathrm{DC}}$ as a function of reciprocal temperature $1 / T$.

gaps between clusters. Figure 4 displays the data for a $1.25 \mathrm{vol} \%$ CB-EA sample as a function of $1 / T$. Over the temperature range used ( 145 to $345 \mathrm{~K}$ ), $\sigma_{\mathrm{DC}}$ can be accurately described $\left(R^{2}=0.9855\right)$ with $T_{0}=0 \mathrm{~K}$ and $T_{1}=226 \mathrm{~K}$, indicating essentially Arrhenius-type behavior. It seems that the $T$-range used is too limited to discern any curvature in a $\ln \sigma_{\mathrm{DC}}$ versus $1 / T$ plot with some accuracy and that a much lower temperature is required to do so. A similar conclusion was obtained before $[9,10]$. Alternative fits using variable range hopping models $[27,28]$ in which $\ln \sigma_{\mathrm{DC}}$ versus $T^{-\gamma}$ with exponent $\gamma=1 / 4$ or $1 / 2$ should provide, if obeyed, a linear relationship, while they resulted in somewhat curved plots $\left(R^{2}=0.9812\right)$. Altogether this indicates that it is virtually impossible to establish the presence of non-ohmic contacts between conductive particles by conventional macroscopic measurements; here, conventional means using liquid nitrogen temperature as the lower limit and an upper limit of, say, $150{ }^{\circ} \mathrm{C}$ typically the maximum temperature allowed for polymers. This may have been clear already from the fact that the $I-V_{\text {ave }}$ curves for $10 \times 10 \mu \mathrm{m}^{2}$ areas are already linear.

\section{General considerations}

Within a general structure-property relationship point of view, one important aim for conductive particulate composites is to be able to determine the conductivity of such a composite from its microstructure. For these composites, the conductive behavior is codetermined by the percolation pathways and the nature of the contacts between the particles. The former has been described many times using percolation theory, albeit generally using thermoplastic matrices with limited attention for the influence of crosslinking on the formation of such pathways [20, 29]. For the latter, when in the literature composite mesoscopic models are discussed, the nature of the contacts is generally considered to be ohmic, which this paper shows, at least for the $\mathrm{CB}$ composites used here, not to be true. Moreover, nonohmic contacts have been discussed in the literature, see, e.g., refs. [26, 30, 31]. Hence, together with the admittedly limited results showing a variety of type of contacts, this implies that making the approximation of ohmic contacts only is not warranted. Considering the option to model $I-V$ curves, either macroscopically or in C-AFM, it will be clear that full details on the complete path between the one electrode (or tip) and the other electrode are required, which so far have not been obtained. Simulations with an effective (constant) contact resistance, taking the various pathways into account as done for carbon nanotube composites [32], will thus be (highly) approximate, as shown in this paper for CB composites, because the contact nature (and area) will vary considerably. We note that papers dealing with the electrical conduction of carbonaceous particulate polymer composites (see, e.g., refs. [9, 10, 29, 33]) generally do not discuss the variable behavior of the contacts at all.

As indicated in the introduction, the main experimental problem to realize reconstructed 3D images by combination of C-AFM and ultramicrotoming is to obtain at least 20-30 good quality C-AFM images on a subsequent series of surfaces where each surface is obtained by successfully removing a 10-20-nm-thick slice. As such experiments are not only time-consuming but also quite tedious, at this stage, we performed experiments mainly for samples with $1.25 \%$ CB. However, we believe that for comparison of the samples, 2D C-AFM images are sufficient, if we assume uniform isotropic structure of all samples. In a forthcoming publication, we plan to present a detailed study of the volume structure in these samples with the tomo-C-AFM technique, in particular by using local $I_{\text {ave }}-V$ curves and nanoimpedance measurements.

\section{Conclusions}

The AC conductivity of carbon black-epoxy-amine (CB-EA) composites has been characterized by a crossover at frequency $f_{\mathrm{c}}$ from a frequency- 
independent $\sigma_{\mathrm{DC}}$ to the frequency-dependent part, particularly on samples with $\mathrm{CB}$ concentration higher than $0.5 \mathrm{vol} \%$. The relationship $f_{\mathrm{c}}=f_{\xi}$ was confirmed, where $\xi$ is the largest fractal length scale. The frequency-dependent conductivity for $f>f_{c}$ thereby can be attributed to the charge diffusion within fractal clusters. The $z$ exponent obtained from fitting $f_{\mathrm{c}} \sim$ $\sigma_{\mathrm{DC}}^{z}$ is 1.14 , which is significantly lower than the values obtained from systems formed through random percolation and diffusion-limited cluster aggregation. Combined with a $t$-value of 2.9 obtained from DC conductivity measurement $\left(\sigma_{\mathrm{DC}-}\right.$ $\left.\sim\left|p-p_{\mathrm{c}}\right|^{t}\right)$, and 3.0 from AC conductivity measurement $\left(d_{\mathrm{rw}}=2+\theta=2+(t-\beta) / v\right)$, charge tunneling between conductive clusters is likely to occur for $f<f_{\mathrm{c}}$. This study done by using conductive atomic force microscopy (C-AFM) directly shows that only part of the clusters in the composite contribute to the overall conductivity, as inferred indirectly for the behavior of other carbon-based composites $[18,19,34]$. The conductivity measurements performed on individual clusters displayed both linear and nonlinear $I-V$ behavior, confirming the conductive contribution of electron tunneling effects between the clusters. Moreover, the 3D reconstructed images offer direct visual evidence that the percolation network of these materials consists of both a lowconductivity part, in which conductivity is controlled by tunneling, and a high-conductivity part, which shows ohmic electrical properties. Nevertheless, conventional temperature-dependent macroscopic conductivity measurements still lead to Arrheniustype behavior for the conductivity despite the presence of these non-ohmic contacts.

\section{Acknowledgements}

The authors thank Dr. A. Efimov for his help with zoomed 3D data reconstruction and the Dutch Polymer Institute for financial support (DPI Project \#617).

\section{Compliance with ethical standards}

Conflict of interest The authors declare that they have no conflict of interest.

Open Access This article is licensed under a Creative Commons Attribution 4.0 International License, which permits use, sharing, adaptation, distribution and reproduction in any medium or format, as long as you give appropriate credit to the original author(s) and the source, provide a link to the Creative Commons licence, and indicate if changes were made. The images or other third party material in this article are included in the article's Creative Commons licence, unless indicated otherwise in a credit line to the material. If material is not included in the article's Creative Commons licence and your intended use is not permitted by statutory regulation or exceeds the permitted use, you will need to obtain permission directly from the copyright holder. To view a copy of this licence, visit http://creativecommons.org/licen ses/by/4.0/.

\section{References}

[1] Lin S, Shih H, Mansfeld F (1992) Corrosion protection of aluminum-alloys and metal matrix composites by polymercoatings. Corros Sci 33:1331-1349

[2] Shaw SJ (1975) Epoxy resin adhesives. In: Ellis B (ed) Chemistry and technology of epoxy resins, vol 1. Black Academic and Professional, Glasgow, pp 206-255

[3] Twite RL, Bierwagen GP (1998) Review of alternatives to chromate for corrosion protection of aluminum aerospace alloys. Progr Org Coat 33:91-100

[4] Meis NNAH, van der Ven LGJ, van Benthem RATM, de With G (2014) Extreme wet adhesion of a novel epoxyamine coating on aluminum alloy 2024-T3. Progr Org Coat 77:176-183

[5] Licari JJ (2003) Chemistry and properties of polymer coatings. In: Licari JJ (ed) Coating materials for electronic applications: polymers, processing, reliability, testing (materials and processes for electronic applications), vol 1. Noyes Publications/William Andrew Inc., New York, pp 65-200

[6] Adriaanse LJ, Reedijk JA, Teunissen PAA, Brom HB, Michels MAJ, Brokken-Zijp JCM (1997) High-dilution carbon-black/polymer composites: hierarchical percolating network derived from $\mathrm{Hz}$ to $\mathrm{THz}$ ac conductivity. Phys Rev Lett 78:1755-1758

[7] Michels MAJ, Brokken-Zijp JCM, Groenewoud WM, Knoester A (1989) A systematic study of percolative network formation and effective electric-response in low-concentration-carbon-black polymer composites. Phys A 157:529-534

[8] Sumita M, Asai S, Miyadera N, Jojima E, Miyasaka K (1986) Electrical-conductivity of carbon-black filled ethylene-vinyl acetate copolymer as a function of vinyl-acetate content. Colloids Polym Sci 264:212-217 
[9] Barrau S, Demont P, Peigney A, Laurent C, Lacabanne C (2003) DC and AC conductivity of carbon nanotubes - polyepoxy composites. Macromolecules 36:5187-5194

[10] Sandler J, Shaffer MSP, Prasse T, Bauhofer W, Schulte K, Windle AH (1999) Development of a dispersion process for carbon nanotubes in an epoxy matrix and the resulting electrical properties. Polymer 40:5967-5971

[11] Roldughin VI, Vysotskii VV (2000) Percolation properties of metal-filled polymer films, structure and mechanisms of conductivity. Prog Org Coat 39:81-100

[12] Psarras GC (2007) Charge transport properties in carbon black/polymer composites. Polym Phys 45(18):2535-2545

[13] Strumpler R, Glatz-Reichenvach J (1999) Conducting polymer composites. J Electroceram 3:329-346

[14] Zallen R (1983) The physics of amorphous solids. Wiley, Hoboken, p 135

[15] Stauffer D, Ahorony A (1987) Introduction to percolation theory, 2nd edn. Taylor \& Francis, London

[16] Magerle R (2000) Nanotomography. Phys Rev Lett 85:2749-2752

[17] Alekseev A, Efimov A, Loos J, Matsko N, Syurik J (2014) Three-dimensional imaging of polymer materials by scanning probe tomography. Eur Pol J 52:154-156

[18] Alekseev A, Efimov A, Lu KB, Loos J (2009) Three-dimensional electrical property mapping with nanometer resolution. Adv Mater 21:4915-4919

[19] Alekseev AM, Efimov AE, de With G (2018) Reconstruction of volume structure of carbon-based conductive polymer composites. IOP Conf. Ser. 443:012002(1-5)

[20] Wu T, Foyet A, Kodentsov A, van der Ven LGJ, van Benthem RATM, de With G (2019) Curing and percolation for carbon black-epoxy-amine nanocomposites. Comp Sci Technol 181:1076721-8

[21] Li W, Schwartz RW (2006) Ac conductivity relaxation processes in $\mathrm{CaCu}_{3} \mathrm{Ti}_{4} \mathrm{O}_{12}$ ceramics: grain boundary and domain boundary effects. Appl Phys Lett 89:242906(1-3)

[22] Gefen Y, Aharony A, Alexander S (1983) Anomalous diffusion on percolating clusters. Phys Rev Lett 50:77-80

[23] Stauffer D (1979) Scaling theory of percolation clusters. Phys Rep 54:1-74
[24] Jäger KM, McQueen DH, Tchmutin IA, Ryvkina NG, Kluppel M (2001) Electron transport and ac electrical properties of carbon black polymer composites. J Phys D Appl Phys 34:2699-2707

[25] Meakin P, Majid I, Havlin S, Stanley HE (1984) Topological properties of diffusion limited aggregation and clustercluster aggregation. J Phys A: Math Gen 17:L975-L981

[26] Sheng P (1980) Fluctuation-induced tunneling conduction in disordered materials. Phys Rev 21:2180-2195

[27] Mott NF (1993) Conduction in non-crystalline materials, 2nd edn. Oxford University Press, New York

[28] Shklovskii BI, Efros AL (1984) Electronic properties of doped semiconductors. Springer, Berlin

[29] Kosmidou ThV, Vatalis AS, Delides CG, Logakis E, Pissis P, Papanicolaou GC (2008) Structural, mechanical and electrical characterization of DGEBA TETA $\backslash \mathrm{CB}$ nanocomposites. eEXPRES Pol Lett 2:364-372

[30] Sichel EK, Sheng P, Gittleman JI, Bozowski S (1981) Observation of fluctuation modulation of tunnel junctions by applied ac stress in carbon polyvinylchloride composites. Phys Rev B 24:6131-6134

[31] Meier JG, Mani JW, Klüppel M (2007) Analysis of carbon black networking in elastomers by dielectric spectroscopy. Phys Rev B 75:054202(1-10)

[32] Gnanasekaran K, Grimaldi C, de With G, Friedrich H (2019) A unified view on nanoscale packing, connectivity, and conductivity of CNT networks. Adv Funct Mater 2019:1807901(1-6)

[33] Boukheir S, Len A, Füzi J, Kenderesi V, Achour ME, Éber N, Costa LC, Queriagli A, Outzirhit A (2016) Structural characterization and electrical properties of carbon nanotubes/epoxy polymer composites. Appl Pol Sci 2016:44514(1-8)

[34] Bârsan OA, Hoffmann GG, van der Ven LGJ, de With G (2016) Single-walled carbon nanotube networks: the influence of individual tube-tube contacts on the large-scale conductivity of polymer composites. Adv Funct Mater 2016:4377-4385

Publisher's Note Springer Nature remains neutral with regard to jurisdictional claims in published maps and institutional affiliations. 